\title{
Spectral compression of an all-normal dispersion fiber laser
}

\author{
Wan-Tien Chao, Hung-Wen Chen, Hsiu-Po Chuang, Shang-Da Yang, and Chen-Bin Huang* \\ Institute of Photonics Technologies, National Tsing Hua University, Hsinchu 30013, Taiwan \\ Email:*robin@ee.nthu.edu.tw
}

\begin{abstract}
Asbtract The feasibility to achieve large-scale spectral compression of an all-normal dispersion fiber laser in a dispersion-increasing fiber is studied both numerically and experimentally. Experimentally, a record-high spectral compression ratio of 46.7 is achieved.
\end{abstract}

\section{Introduction}

In spectroscopic applications, laser sources with high spectral brightness (power density) are essential in enhancing the signal-to-noise ratio and the reduction of the measurement time. However, typical fs and supercontinuum sources suffer from low spectral brightness due to their inherent wide bandwidth. An interesting solution is to perform external laser spectral compression to effectively enhance the spectral brightness through the redistribution of the energy into a narrow user-desired spectral window. Such spectral narrowing effect was first explained for a negatively-chirped optical pulse propagating in a normal dispersion optical fiber [1]. Chirped optical pulses within standard single-mode fiber [2] and photonic crystal fiber [3,4] were also capable of achieving spectral compression with a moderate spectral compression ratio (below 7). A comb-profiled fiber was used to demonstrate quasi-adiabatic soliton spectral compression [5]. On the other hand, true adiabatic soliton spectral compression using a dispersion-increasing fiber (DIF) was experimentally demonstrated using a soliton fiber laser [6]. Recently, the waveform dependence (parabolic pulses) over the spectral compression ratio within a normal dispersive photonic crystal fiber was addressed [3]. In this paper, we first present numeical analysis on the feasibility in achieving largescale spectral compression ratio within a DIF with linear dispersion ramp. Spectral compressions using same input full-width half-maximum (FWHM) pulse duration, but different waveforms such as sech, Gaussian, parabolic, and dissipative soliton derived from an all-normal dispersion (ANDi) laser [7] are compared. Our numerical results indicate that the ANDi laser can provide the largest compression ratio (greater than 40). Moreover, such large-scale spectral compressions are independent of the signs of the initial pulse chirp applied to input pulses. Experimentally, a record-high spectral compression ratio of 46.7 achieved by launching 160 fs positively-chirped ANDi laser pulses into a DIF.

\section{Numerical analysis}

The feasibility over waveform-dependent spectral compression within the DIF is numerically assessed. The calculations are performed by solving the generalized nonlinear Schrödinger equation using the split-step Fourier method with 2000 computational steps. The anomalous dispersive DIF is modeled using a linear dispersion ramp of 0.6 to $13.5 \mathrm{ps} / \mathrm{nm} / \mathrm{km}$ from the input to the output, an input dispersion slope of $0.07 \mathrm{ps} / \mathrm{nm}^{2} / \mathrm{km}$, a loss coefficient of $0.4 \mathrm{~dB} / \mathrm{km}$, along with the nonlinear and Raman coefficients of $3.5(\mathrm{~W}-\mathrm{km})^{-1}$ and $3 \mathrm{fs}$, respectively.
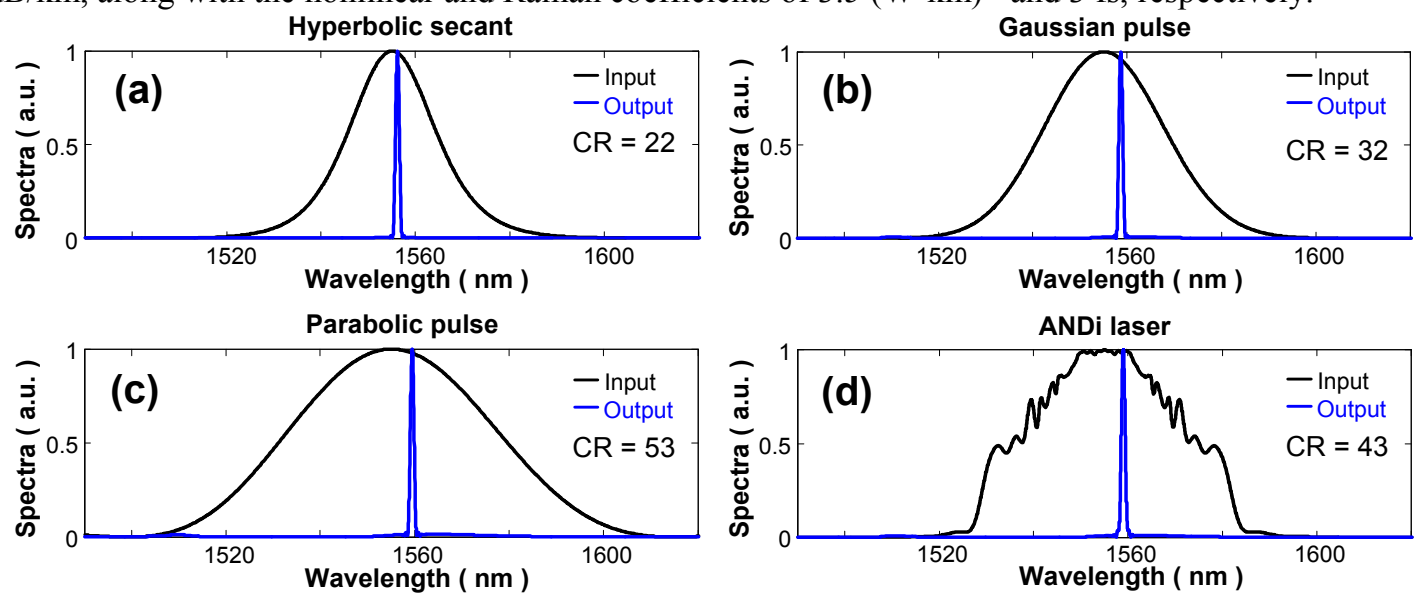

Fig. 1 Spectral compression using 121 fs FWHM input pulses of (a) hyperbolic secant, (b) Gaussian, (c) parabolic pulses. (d) Spectral compression using ANDi laser. The compression ratios (CR) are indicated in each figure.

Figures 1(a-c) show the input and spectrally compressed spectra when 121 fs FWHM duration hyperbolic secant, Gaussian and parabolic pulses are launched into the DIF, respectively. Here, the spectral compression ratio 
(CR) is defined by the ratio of the FWHM spectral bandwidth of the input spectrum to the output spectrum. Our results show the parabolic pulse can provide a highest CR of 53, followed by Gaussian pulse with a CR of 32, while the hyperbolic secant pulse provided the lowest CR of 22 . While the parabolic pulse can provide the highest CR value, the generation of such pulse often requires additional amplitude pulse shaping outside the laser cavity. Therefore, a pulse shape that can be derived directly from a laser cavity while capable of providing large CR value is of essential value. Figure 1(d) shows the spectral compression result using pulse derived from an ANDi laser. The spectrum and the waveform are obtained by solving the cubic-quintic Ginzburg-Landau equation [7]. The ANDi laser pulse can provide a high compression ratio of 43 .

\section{Experimental results}

Figure 2 shows the schematics of the experimental setup. The optical source is an ANDi laser with $8 \mathrm{MHz}$ repetition frequency [8], capable of supporting 139 fs transform-limited pulses. The DIF parameters are in accordance with our numerical studies. A segment of single mode fiber (SMF) is used to compensate the normal dispersion of the laser output pulse. The ten percent port of an optical coupler is used to monitor the power launched into the DIF. The ninety percent port is connected to a $1-\mathrm{km}$ length dispersion-flattened DIF. The spectrally compressed optical spectra after the DIF are measured using an optical spectrum analyzer (OSA).

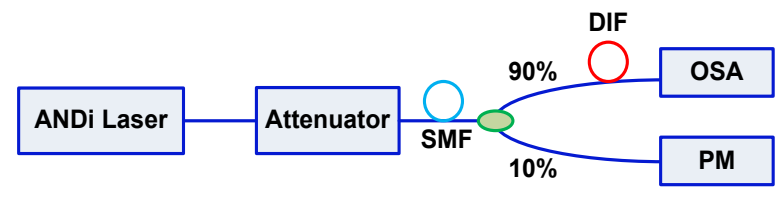

Fig. 2 Schematic experimental setup: SMF, single mode fiber; DIF: dispersion-increasing fiber; PM, power meter; OSA, optical spectrum analyzer.

Figure 3(a) shows the experimental spectrally compressed optical spectrum after the DIF (blue line) plotted against the initial ANDi laser spectrum (black line) with an input average power of $62.6 \mu \mathrm{W}$. The input pulse is near transform-limited. The FWHM bandwidth of spectrum after DIF is $0.93 \mathrm{~nm}$, giving a record high spectral compression ratio of 46.7. Figure 3(b) shows the experimental and the calculated compressed optical spectra. The experiment and calculation are in good agreements.
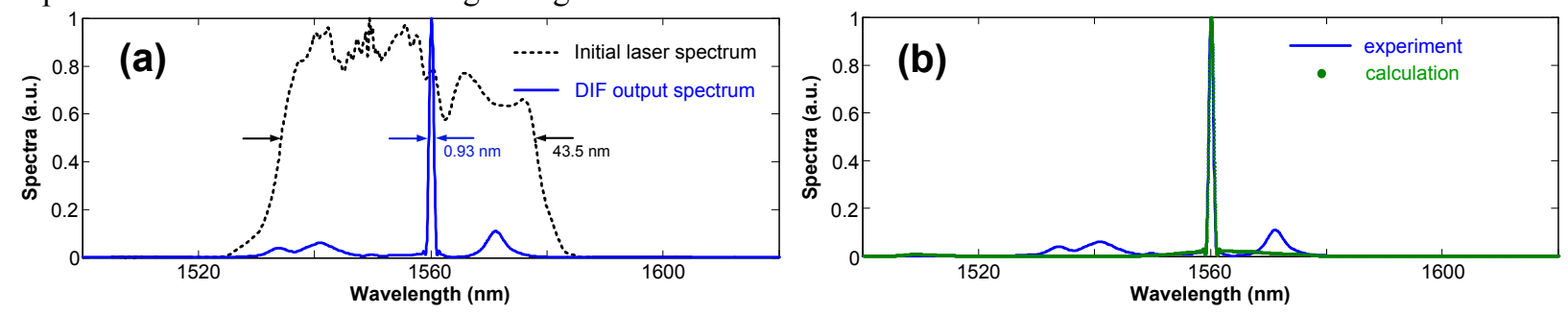

Fig. 3 (a) Experimental DIF output spectrum giving a spectral compression ratio of 46.7. (b) Experimental (solid blue curve) and calculated (green dot curve) compressed spectra.

\section{Conclusion}

In summary, the feasibility in yielding large spectral compression using an ANDi laser is numerically studied. A record-high spectral compression ratio of 46.7 is experimentally achieved by launching positively chirped optical pulses derived from an ANDi fiber laser into a 1-km dispersion-increasing fiber. This work was supported by the National Science Council in Taiwan under grant NSC 100-2112-M-007-007-MY3, 100-2221-E-007-093-MY3 and by National Tsing Hua University grant 101N2081E1.

\section{References}

[1] S. A. Planas, N. L. Pires Mansur, C. H. Brito Cruz, and H. L. Fragnito, Opt. Lett. 18, 699 (1993).

[2] B. R. Washburn, J. A. Buck, and S. E. Ralph, Opt. Lett. 25, 445 (2000).

[3] E. R. Andresen, J. M. Dudley, D. Oron, C. Finot, and H. Rigneault, Opt. Lett. 36, 707 (2011).

[4] A. B. Fedotov, A. A. Voronin, I. V. Fedotov, A. A. Ivanov, and A. M. Zheltikov, Opt. Lett. 34, 662 (2009).

[5] N. Nishizawa, K. Takahashi, Y. Ozeki, and K. Itoh, Opt. Express 18, 11700 (2010).

[6] H.-P. Chuang and C.-B. Huang, Opt. Lett. 36, 2848 (2011).

[7] W. H. Renninger, A. Chong, and F. W. Wise, Phys. Rev. A 77, 023814 (2008).

[8] H.-W. Chen and S.-D. Yang, Proc. IEEE Photonics Conference, WJ3, 2012. 